\title{
Deliberate Limits to Arbitrage
}

\author{
Igor Makarov*and Guillaume Plantin ${ }^{\dagger \ddagger}$
}

February 23, 2012

\begin{abstract}
This paper develops a model in which arbitrageurs are collectively unconstrained, but may still prefer to incur individual limits to arbitrage rather than make full use of their combined resources. These deliberate limits arise because the communication of an arbitrage position reveals the underlying idea, which creates future competition in the absence of relevant property rights. We allow arbitrage opportunities to vary along two dimensions: the ease with which they can be identified and the speed at which they mature. We find that deliberate limits to arbitrage arise for opportunities in the mid-range of the maturity dimension. This range widens when the opportunities are easier to find. Our results thus offer a set of theoretical predictions about the arbitrage trades that are likely to exist in the market.
\end{abstract}

${ }^{*}$ London Business School. E-mail: imakarov@london.edu.

${ }^{\dagger}$ Toulouse School of Economics and CEPR. E-mail: guillaume.plantin@tse-fr.eu.

${ }^{\ddagger}$ We thank Ulf Axelson, Bruno Biais, Mike Chernov, James Dow, Daniel Ferreira, Christopher Hennessy, Peter Kondor, Augustin Landier, Oleg Rytchkov, Dimitri Vayanos and seminar participants at LBS, LSE, and the third Oxford-Man Institute Hedge Fund Conference for very helpful comments. 


\section{Introduction}

Textbook asset pricing theory suggests that portfolios with similar revenues should have similar prices. However, many real-world price patterns do not comport well with this prediction. ${ }^{1}$ A large body of finance research seeks to account for such anomalies by extending the textbook theory to include limits to arbitrage, since arbitrage activity in practice is subject to potentially significant constraints that are ignored by canonical asset pricing models.

Limits to arbitrage models all make the assumption that there is a "separation of brains and resources" in capital markets. More precisely, they build on a vision of markets in which only a small number of elite investors - the arbitrageurs - have the knowledge and skills to identify and exploit pricing anomalies. Because arbitrage activity requires more capital than the amount owned by these arbitrageurs, they must tap the general population of investors for additional resources. However, the potential value from an arbitrage trade cannot be entirely pledged to these investors as they lack the knowledge, information, or cognitive ability required to properly assess the arbitrageurs' projects. As a result, arbitrageurs may face financial constraints and pricing anomalies may persist. In a seminal paper, Shleifer and Vishny (1997) suggest that rational speculation may even increase market volatility in the presence of these constraints, contrary to the classical arguments of Friedman (1953).

In light of the evolution of financial markets in recent decades, the assumption that there is too little capital in the hands of sophisticated arbitrageurs seems, at least, questionable. The financial sector has grown considerably, attracting a lot of high-quality and well-compensated human capital (e.g. Goldin and Katz, 2008 and Philippon and Reshef, 2011). Some observers have even called this growth excessive. Accordingly, the share of finance professionals in top U.S. income levels has increased significantly (Kaplan and Rauh, 2010). Deregulation and the rise of the private equity and hedge fund industries have greatly reduced institutional constraints for financially sophisticated agents. Overall, it can no longer be taken for granted that investors with the ability to identify asset pricing anomalies suffer from an aggregate shortage of resources.

This paper argues that limits to arbitrage may still arise when aggregate resources are plentiful. We develop a model with collectively unconstrained arbitrageurs who may nonetheless limit their individual activity rather than collaborate with their competitors.

Our theory is based on the nature of the knowledge required to identify and exploit

\footnotetext{
${ }^{1}$ See, for example, Barberis and Thaler (2003), Lamont and Thaler (2003), and Gromb and Vayanos (2010) for surveys of the empirical evidence.
} 
arbitrage opportunities. This knowledge has three important properties. First, it can be kept secret (at least in the short run). If an arbitrageur does not communicate beyond minimum disclosure requirements, other arbitrageurs will find it difficult to replicate or reverse-engineer his trades. Second, communication of an arbitrage position cannot be unbundled from communication of the underlying knowledge. If an arbitrageur reveals the details of an arbitrage position to an equally sophisticated arbitrageur, the latter can uncover the underlying strategy and use it to trade in the future. Third, arbitrage knowledge cannot be patented. Taken together, these three features imply that arbitrageurs face a trade-off between the financial constraints associated with secrecy, and the future competition created by coordination with other arbitrageurs.

To better understand this trade-off, we develop a model with recurrent arbitrage opportunities in a financial market. Opportunities can be located by a particular arbitrageur only if he owns a search technology. While arbitrageurs have sufficient collective financial wealth to seize every open opportunity, each arbitrageur may be individually constrained. Thus a constrained arbitrageur with a search technology may find it worthwhile to contact an unconstrained arbitrageur in order to profit from an opportunity that he cannot exploit himself. However, in communicating the opportunity to the potential buyer, he also communicates the search technology which the buyer may then use.

Such communication may take several forms in practice. For example, after identifying a mispricing, the arbitrageur may first take a position and then "talk up his own book" or recommend the trade to other speculators, hoping that they will copy his position. By doing so, they narrow the price gap and allow the initial arbitrageur to realize a profit. Many high-profile investors, including Warren Buffet and Bill Gross, are believed to follow this strategy on occasion.

Similarly, in our model, an arbitrageur can realize the profit from an existing position by selling it to another arbitrageur for an amount greater than cost, then redeploying the funds to a newly-found opportunity. Such a sale occurs only if the cost of increased competition for similar future opportunities is less than or equal to the sale price. The sale price is capped, in turn, by the potential buyer's outside option to reject the offer and seek other open opportunities, using the search technology acquired in communication with the seller. When the expected cost of future competition is larger than the profit from selling an existing position, arbitrageurs will choose to remain opaque and constrained rather than share the benefits from optimally deploying their aggregate resources. The economy thus displays deliberate limits to arbitrage.

We find that deliberate limits are more likely to occur when arbitrage opportunities 
are neither too quick nor too slow to mature, because the horizon of an arbitrage trade has an ambiguous effect on the incentive to share arbitrage business. Consider what happens when opportunities take a longer time to pay off (all else being equal). On the one hand, the costs of future competition are more remote, occurring only after the current opportunities mature. Knowledge-sharing from the sale of the already-established arbitrage position is therefore less problematic. On the other hand, although the costs are remote, they are also more important when they occur. Market congestion caused by competing arbitrageurs is high in this case because arbitrageurs exhaust open trading opportunities more frequently. This renders knowledge sharing more costly. Which effect prevails depends on the parameters of the model.

We also show that when arbitrage opportunities are easier to find, deliberate limits arise for a wider range of maturities. This happens because a more efficient search technology simultaneously makes it harder for the initial arbitrageur to sell a position for its full value and increases the effect of future competition.

Our results thus indicate which arbitrage trades are likely to exist in the market. In particular, our model suggests that unique ideas, which cannot be implemented many times over, should be less prone to deliberate limits to arbitrage. For such one-off trades, it is in the interests of the arbitrageurs who originally establish the positions to "talk up their books", as this does not come at the cost of fiercer competition on similar future opportunities.

Our paper is related to the literature on limits to arbitrage pioneered by Dow and Gorton (1994) and Shleifer and Vishny (1997) and recently surveyed in Gromb and Vayanos (2010). Newer contributions to this literature emphasize the potentially destabilizing role of constrained arbitrageurs. Kondor (2009) shows how limited arbitrage capital may cause prices to diverge as a result of arbitrageurs' optimal timing of trades. Brunnermeier and Pedersen (2009) and Gromb and Vayanos (2011) show how interim losses lead arbitrageurs to deleverage, thereby amplifying their losses and triggering further deleveraging. He and Krishnamurthy (2011) rationalize the capital constraints that generate such deleveraging spirals as second-best contracts in the presence of moral hazard. Our contribution to this literature is to offer foundations for the "separation of brains and resources" concept on which all of these papers build. We show how the lack of property rights on arbitrage ideas endogenously generates the informational asymmetries and capital constraints that lie at the heart of these models.

This paper also contributes to the general literature on the sale of ideas. According to the Arrow information paradox (Arrow, 1962), an idea must be communicated in order to be sold at a positive price, but the potential buyer has no reason to pay anything once he becomes aware of the idea. It is therefore difficult to sell ideas at a 
fair price. Following seminal work by Bhattacharya and Ritter (1983), a large literature has studied solutions to the Arrow information paradox (see, for example, Anton and Yao (2002) and references therein). Our contribution is to offer a simple infinite-horizon setup in which the intertemporal trade-off between relaxing capital constraints through the sale of ideas and remaining secretive but constrained can be studied.

Finally, our approach is close to a contribution by Kondo and Papanikolaou (2005). They study a situation in which the communication of information between sophisticated arbitrageurs is feasible in principle, but limited in practice by the fact that the receiver cannot commit to avoid front-running the sender. Our approaches are complementary. We analyse knowledge sharing in an environment where agents interact through simple spot trades and lack any commitment power. Conversely, in Kondo and Papanikolaou (2005), knowledge sharing cannot occur without repeated interactions and commitment power. They show that knowledge sharing occurs only when the seller of ideas can credibly commit to punish the deviations of the buyer.

\section{$1 \quad$ Model}

Time is continuous. There is a single consumption good used as the numéraire. There are $N$ agents who seek to identify and exploit arbitrage opportunities in a financial market. These $N$ arbitrageurs are risk-neutral, infinitely-lived, and discount future consumption at the instantaneous rate $r>0$. There are exactly $M$ arbitrage opportunities at each date, where $M \leq N$. Each opportunity pays off at a random date that occurs according to a Poisson process with intensity $r x$, where $x>0$. At this final date, a new opportunity opens up to replace the expired one, hence the constant number of opportunities. Arbitrage opportunities are positive-NPV trades with the following exogenous cash flows. Exploiting an open opportunity requires a fixed initial investment of one unit. This initial investment can take place at any date before the opportunity matures, and yields $1+R$ at maturity, where $R>1 / x$.

Arbitrageurs cannot readily identify arbitrage opportunities, but rather need to search for them. An arbitrageur who does not own the search technology cannot find arbitrage opportunities. An arbitrageur who owns it can search simultaneously for all open opportunities. He uncovers a given opportunity according to a Poisson process with intensity $r y$, where $y>0$. This search technology captures that the $M$ arbitrage opportunities represent different implementations of the same broad arbitrage idea (e.g., convertible bond arbitrage, merger arbitrage, etc.). Owning the search technology means knowing how to implement this idea in principle, and searching means identifying market situations in which this idea can be effectively implemented. 
All Poisson processes are assumed to be mutually independent.

We assume that arbitrageurs are subject to capacity constraints. Each arbitrageur can gain exposure to at most one opportunity at a time. While arbitrageurs are individually constrained, collectively they are not since $M \leq N$. An arbitrageur who has identified more than one opportunity and has already invested in one of them may sell an opportunity to an arbitrageur with no current investment. In order to sell an arbitrage opportunity that he has identified, an arbitrageur needs to explain it to his potential buyer. Crucially, after a given arbitrage opportunity has been explained to him, the potential buyer is not only aware of this particular opportunity, but he also owns the search technology if he did not already. Further, he then has a free hand at exploiting this opportunity and his search technology as he sees fit.

\subsection{Some Comments on the Assumptions}

Before analyzing the model, it is worthwhile explaining the role of three assumptions that the reader may find unusual.

First, the fixed capacity of arbitrageurs captures in the simplest form the fact that arbitrageurs are unable to fully exploit all their available opportunities with the funds at their disposal. This simple specification of the capacity constraints eliminates some interesting dynamic aspects of limits of arbitrage. For example, in a richer model, the track record of an arbitrageur could affect his current and future capacity constraints. Further, these constraints can be mitigated by using an optimal capital accumulation policy. While these aspects of limits of arbitrage are interesting in their own right, they are not essential to our argument. Therefore, we prefer to leave them out so as to make our main point more clearly and more simply.

Second, the fixed scale of each arbitrage opportunity is a stark form of decreasing returns to scale in arbitrage. Decreasing returns to arbitrage are arguably realistic. If arbitrage opportunities were perfectly scalable, then mispricing would persist no matter how much capital is used to profit from them, which seems unlikely. Decreasing returns to scale make mutually profitable trades possible in our model. If each opportunity was perfectly scalable, then an arbitrageur would not be willing to purchase an established position at more than cost since he would have the option to scale it up himself. With fixed opportunities, an arbitrageur who has first established a position obtains a rent that he can partially sell to other arbitrageurs. This comes, however, at the cost of competing with them in the future.

Such trades of fixed-size opportunities capture in a stylized but simple way the following real-world trade-off. After having identified a mispricing and taken a position, 
an arbitrageur benefits from having other speculators copying his position in practice, because their price impact narrows the price gap further, allowing him to realize a profit. In our model, such a profit is realized by selling a fixed-size opportunity at more than its unit cost. As in our model, the gains made on a current position by the entry of "copycat funds" comes at the cost that this creates competition for future similar and yet unseized opportunities.

Finally, we implicitly rule out the possibility that an arbitrageur enters into a delegated investment relationship with a fellow arbitrageur, whereby he promises future returns without revealing what he does. The contracting problem would be nontrivial since the existence of an opportunity and its payoff dates are privately observed. Studying such an arrangement would amount to an analysis of a standard limits-to-arbitrage problem in which optimal contracts mitigate informational asymmetries. Since our goal is precisely to explain why such informational asymmetries endogenously arise in the first place, we find it natural to study the situation in which arbitrageurs face the binary choice of information sharing versus opaqueness and autarky. This restriction works against the occurrence of endogenous limits of arbitrage. Our assumed alternative to full information sharing - autarky - is more costly to arbitrageurs than optimal delegated investment under informational asymmetry would be.

\subsection{One Arbitrage Opportunity, One Arbitrageur}

We first consider a simple situation where there is only one arbitrage opportunity and one arbitrageur $(M=N=1)$ who owns the search technology. The arbitrageur can be in two possible states. Either he is searching for the current opportunity, or he has identified it, and remains invested in it until it matures. Denote $W_{0}$ and $W_{1}$ his utilities in these respective states. We have

$$
\begin{aligned}
& r W_{0}=r y\left(W_{1}-1-W_{0}\right), \\
& r W_{1}=r x\left(W_{0}+R+1-W_{1}\right)
\end{aligned}
$$

which yields

$$
\begin{aligned}
& W_{0}=\frac{y}{1+x+y}(x R-1), \\
& W_{1}=1+\frac{1+y}{1+x+y}(x R-1) .
\end{aligned}
$$

Introducing

$$
W=\lim _{y \rightarrow+\infty} W_{0}=x R-1,
$$


we have

$$
\begin{aligned}
W_{0} & =\frac{y W}{1+x+y}, \\
W_{1} & =1+\frac{(1+y) W}{1+x+y} .
\end{aligned}
$$

The limiting expected present value of future arbitrage opportunities when $y \rightarrow+\infty$, $W$, is simply the value of a perpetuity that pays instantaneously the flow value of arbitrage $r x R-r$. For a finite $y$, this limiting value is discounted with the coefficient

$$
\frac{y}{1+x+y}=\int_{0}^{+\infty} r y e^{-r(1+x+y) t} d t,
$$

which is the expected present value from receiving one dollar if a given arbitrage opportunity is identified before it matures. This captures that a fraction of the opportunities expire before being uncovered and thus are missed by the arbitrageur when $y$ is finite.

\subsection{Two Arbitrage Opportunities, One Arbitrageur}

Consider now the case in which a single arbitrageur searches for two arbitrage opportunities $(M=2, N=1)$. The arbitrageur may now be in three different states. He may either be searching for both opportunities, or, having found and invested in one, he may still be searching for the other one. Finally he may have found the current two opportunities, but have only invested in the first one that he identified because of the capacity constraint. Denote $V_{0}, V_{1}$, and $V_{2}$ the three continuation utilities associated with each respective situation. We have

$$
\begin{aligned}
& r V_{0}=2 r y\left(V_{1}-1-V_{0}\right), \\
& r V_{1}=r x\left(V_{0}+R+1-V_{1}\right)+r y\left(V_{2}-V_{1}\right), \\
& r V_{2}=r x\left(2 V_{1}+R-2 V_{2}\right),
\end{aligned}
$$

which yields

$$
\begin{aligned}
& V_{0}=W \frac{2 y(1+2 x+y)}{(1+x+y)(1+2(x+y))}, \\
& V_{1}=1+W \frac{(1+2 y)(1+2 x+y)}{(1+x+y)(1+2(x+y))}, \\
& V_{2}=1+W \frac{(1+2 y)(1+2 x+y)+x}{(1+x+y)(1+2(x+y))} .
\end{aligned}
$$


Notice that

$$
V_{0}=\left(1+\frac{1+2 x}{1+2 x+2 y}\right) W_{0}>W_{0} .
$$

Compared to the case in which there is only one arbitrage opportunity, the arbitrageur is strictly better off when there are two arbitrage opportunities, in particular because after he reaps the benefit from one opportunity, he can invest right away in the other opportunity if he has spotted it.

\subsection{Two Arbitrage Opportunities, Two Arbitrageurs}

We now consider a situation in which there are two arbitrage opportunities and two arbitrageurs that are both equipped with the search technology $(M=N=2)$. When one arbitrageur identifies two arbitrage opportunities he may sell one of them to the other arbitrageur, so that no identified arbitrage opportunity remains unexploited. Recall that in order to sell an arbitrage opportunity the seller has to explain it first to the potential buyer. Because the potential buyer is free to implement the arbitrage himself without compensating for the tip, an arbitrageur can only sell an arbitrage in which he has already invested.

Suppose for now that such sales take place at a price $P$. We will identify the range for feasible transfer prices below. There are now four possible states in this economy: Either no arbitrageur has found an arbitrage opportunity, or only one of them found and invested in one, or each of them invested in an opportunity. Denote $V_{00}, V_{01}$, $V_{10}$, and $V_{11}$ the associated continuation utilities. In particular, $V_{01}$ is the continuation utility of the arbitrageur who has not found an opportunity while his counterpart has found one, and $V_{10}$ is that of this counterpart. We have

$$
\begin{aligned}
& r V_{00}=2 r y\left(V_{10}-1+V_{01}-2 V_{00}\right), \\
& r V_{01}=r x\left(V_{00}-V_{01}\right)+r y\left(2 V_{11}-P-1-2 V_{01}\right), \\
& r V_{10}=r x\left(V_{00}+1+R-V_{10}\right)+r y\left(2 V_{11}+P-1-2 V_{10}\right), \\
& r V_{11}=r x\left(V_{01}+1+R+V_{10}-2 V_{11}\right),
\end{aligned}
$$


which yields

$$
\begin{aligned}
V_{00} & =\frac{2 y W}{1+x+2 y}, \\
V_{01} & =\frac{2 y W}{1+x+2 y}-\frac{y(P-1)}{1+x+2 y}, \\
V_{10} & =1+\frac{(1+2 y) W}{1+x+2 y}+\frac{y(P-1)}{1+x+2 y}, \\
V_{11} & =1+\frac{(1+2 y) W}{1+x+2 y} .
\end{aligned}
$$

We can see that $V_{00}$ and $V_{11}$ do not depend on the sale price $P$ because each arbitrageur expects future buys and sells to be identically distributed. Notice also that $V_{00}$ and $V_{11}$ are equal to $W_{0}$ and $W_{1}$ respectively when the search parameter is $2 y$ instead of $y$. When arbitrageurs share arbitrage opportunities, it is as if there were two arbitrageurs each specialized in one arbitrage opportunity with a search technology with parameter $2 y$ instead of $y$. Finally, we have

$$
V_{00}<V_{0}
$$

because

$$
\frac{V_{00}}{V_{0}}=\frac{(1+x+y)(1+2 x+2 y)}{(1+2 x+y)(1+x+2 y)}=\frac{1+2 x^{2}+2 y^{2}+4 x y}{1+2 x^{2}+2 y^{2}+5 x y}<1 .
$$

Even though the presence of the second arbitrageur relaxes capacity constraints, an arbitrageur is better off operating on his own from an ex ante perspective. As will be detailed in the next section, there are two costs from sharing opportunities with another arbitrageur. First, each arbitrageur needs in this case to find a new opportunity after his investment pays off. Conversely, an autarkic arbitrageur who has found two opportunities may invest immediately in the second opportunity after the first opportunity matures. Second, there are decreasing returns to search intensity, so that doubling search intensity and splitting up the gains is undesirable.

We now determine the range of feasible transfer prices $P$. Suppose that at a given date, an arbitrageur ("the seller") has invested in one opportunity and just found the other one while the other arbitrageur ("the buyer") has not identified the latter opportunity. Suppose that both believe that future transfers (those that will come after the current one) will take place at some given price $P$. The buyer has two options. He may either buy the current investment of the seller, or he may search himself for the other arbitrage opportunity in which the seller has not been able to invest yet.

Denote $V_{02}$ the continuation utility of the buyer after exercising this latter option. 
Then the maximal price $P_{\max }$ at which the buyer prefers the former option solves

$$
V_{11}-P_{\max }=V_{02}
$$

The continuation value $V_{02}$ solves

$$
r V_{02}=2 r x\left(V_{01}-V_{02}\right)+r y\left(V_{11}-1-V_{02}\right),
$$

or

$$
V_{02}=\frac{2 x V_{01}+y\left(V_{11}-1\right)}{1+2 x+y} .
$$

Thus

$$
P_{\max }=V_{11}-V_{02}=1+\frac{W(1+2 x+2 y)+2 y x(P-1)}{(1+2 x+y)(1+x+2 y)} .
$$

It is easy to see from (1.1) that $P_{\max }$ increases w.r.t. $P$. Thus the maximal price at which sales can take place is obtained when $P=P_{\max }$ in (1.1), or

$$
P_{\max }=1+\frac{W(1+2 x+2 y)}{1+2 x^{2}+3 x y+2 y^{2}+3(x+y)} .
$$

Notice that $P_{\max }>1$.

The minimal price at which the seller is willing to sell his current arbitrage position is such that

$$
V_{11}+P_{\min }-1=V_{20},
$$

where $V_{20}$ is his continuation utility if he decides not to sell his current position. We have

$$
r V_{20}=r x\left(2 V_{10}+R-2 V_{20}\right)+r y\left(V_{11}-V_{20}\right),
$$

or

$$
V_{20}=\frac{x\left(2 V_{10}+R\right)+y V_{11}}{1+2 x+y} .
$$

This yields

$$
P_{\min }=1+V_{20}-V_{11}=1+\frac{x(W+2 y(P-1))}{(1+2 x+y)(1+x+2 y)} .
$$

It is easy to see from $(1.2)$ that $P_{\min }$ increases w.r.t. $P$. Thus the minimal price at which the seller is willing to sell solves $P=P_{\min }$ in (1.2), which yields

$$
P_{\min }=1+\frac{x W}{1+2 x^{2}+3 x y+2 y^{2}+3(x+y)} .
$$


Notice that $P_{\min }>1$, and that

$$
P_{\max }-P_{\min }=\frac{W(1+x+2 y)}{1+2 x^{2}+3 x y+2 y^{2}+3(x+y)}>0 .
$$

Therefore, an arbitrage position can be sold at any given price $P \in\left[P_{\min }, P_{\max }\right]$.

\section{Deliberate Limits to Arbitrage}

The two cases studied in Sections 1.3 and 1.4 prepare the ground to tackle the central question of this paper: When does a constrained arbitrageur prefer to endure limits to arbitrage rather than share his opportunities with other arbitrageurs? To answer this question, we consider again a setting with two arbitrageurs and two arbitrage opportunities $(M=N=2)$.

The difference with section 1.4 is that we now assume that only one of the two arbitrageurs owns the search technology at the outset. We refer to him as the incumbent. This arbitrageur may remain secretive about his activities, in which case he stays in this autarkic situation forever. He may also seek to contact the other arbitrageur to sell him an arbitrage opportunity. After this initial contact, the economy is in the situation of Section 1.4, in which both traders own the search technology. We deem the situation in which the incumbent prefers autarky one of "deliberate limits to arbitrage."

We have seen that $V_{0}>V_{00}$ so that autarky is ex ante preferable. Still, if the incumbent arbitrageur is invested in one opportunity and has found the other one, he may find it worthwhile to sell its current investment at a profit and invest right away in the other opportunity. Again, the incumbent cannot pledge an opportunity that he has found and in which he has not invested yet because the new arbitrageur could just pretend being uninterested and invest in it on his own.

Denote $P_{\text {share }}$ the minimal price at which the incumbent is willing to sell his current arbitrage trade. There will be endogenous deliberate limits to arbitrage if this price is higher than the maximal price $P_{\max }$ derived in Section 1.4 that the new arbitrageur would be willing to pay. Thus deliberate limits to arbitrage occur if and only if

$$
P_{\text {share }}>P_{\max } .
$$

The minimal price at which the incumbent is willing to sell his current arbitrage trade solves

$$
V_{11}-1+P_{\text {share }}-V_{2} \geq 0,
$$


or

$$
P_{\text {share }}=V_{2}-V_{11}+1
$$

Using explicit formulas for $V_{2}, V_{11}$, and $P_{\max }$ computed in Sections 1.3 and 1.4 we have the following proposition:

Proposition 1 There are deliberate limits to arbitrage if and only if

$$
\Phi(x, y)<0
$$

where

$$
\begin{aligned}
\Phi(x, y) & =\varphi_{4}(x) y^{4}+\varphi_{3}(x) y^{3}+\varphi_{2}(x) y^{2}+\varphi_{1}(x) y+\varphi_{0}(x) \\
\varphi_{0}(x) & =-\left(2 x^{4}+7 x^{3}+9 x^{2}+5 x+1\right) \\
\varphi_{1}(x) & =-\left(11 x^{3}+29 x^{2}+25 x+7\right) \\
\varphi_{2}(x) & =4 x^{3}-19 x^{2}-39 x-18 \\
\varphi_{3}(x) & =6 x^{2}-16 x-20 \\
\varphi_{4}(x) & =4 x-8 .
\end{aligned}
$$

In particular, there is sharing of arbitrage knowledge if $x \leq 2$. For each $x>2$, there exists a unique $\psi(x)>0$ such that deliberate limits to arbitrage arise if and only if $y>\psi(x)$.

Proof. See the Appendix.

Notice that whether arbitrage is limited or not depends only on the duration parameters $x$ and $y$, but not on $R$ and therefore nor on the total expected present value of arbitrage. Figure 1 plots the graph of $\psi$ in the plane $(x, y)$.

For small $x$ and/or $y$, the incumbent shares knowledge with the other arbitrageur. Such small $x, y$ correspond to the situation in which only the two currently identified opportunities matter because the discovery and/or exploitation of additional opportunities are very remote. In this situation, knowledge sharing is desirable because it has the immediate benefit that the incumbent can sell the first opportunity at a profit and exploit the second opportunity while the future costs of heightened competition is small.

The graph of $\psi$ has a vertical asymptote $x=2$ and asymptote $x=2 y^{2}$ when $x, y \rightarrow+\infty$. Limits to arbitrage occur only if $y$ is above some minimal value, $y_{\min }$. For a fixed $y \geq y_{\text {min }}$, limits to arbitrage occur if and only if $x$ is neither too small nor too large. That is, it occurs over a subinterval of $(2,+\infty)$. The lower bound of this interval decreases and the upper bound increases as $y$ increases. Thus, an increase 
Figure 1: $\psi(x)$

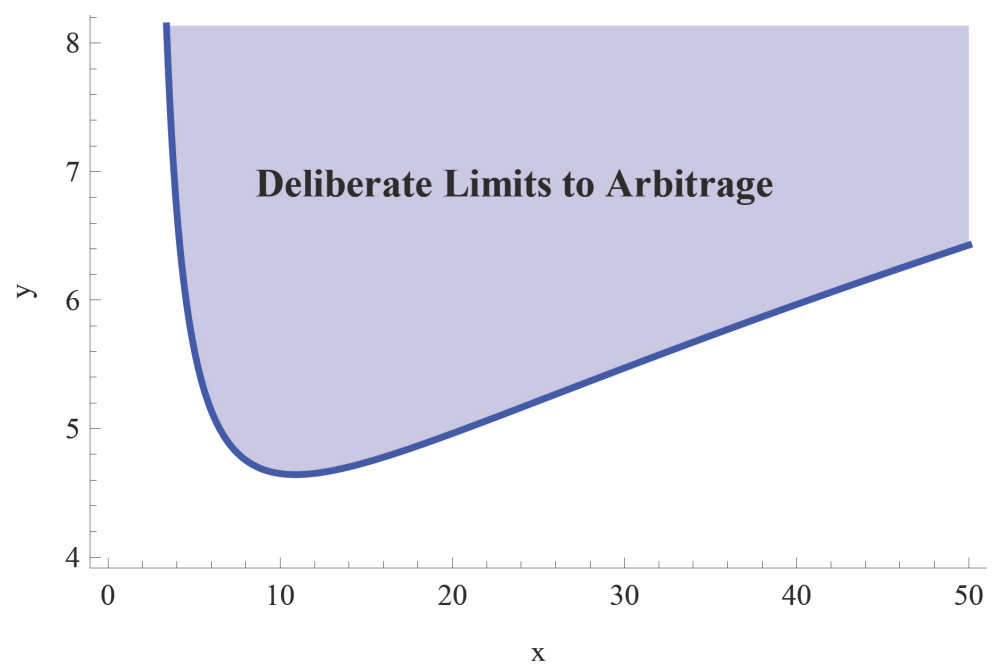

in $y$ makes limits to arbitrage overall more likely while changes in $x$ given $y$ have an ambiguous effect on limits to arbitrage. In order to gain intuition for these results, we study the two limiting cases of the model that correspond to the two asymptotes of $\psi$. We suppose that arbitrage opportunities can be identified quickly $(y \gg 1)$, and study in turn the cases in which they mature slowly and quickly.

\subsection{The Case $y \gg 1, x \approx 2$}

In this case, using formulas derived in Sections 1.3, 1.4 , and 2 one can show that

$$
\begin{aligned}
P_{\max } & =1+\frac{W}{y}+o\left(\frac{1}{y}\right) \\
V_{2} & =1+W+o\left(\frac{1}{y}\right) \\
V_{11} & =1+W-\frac{W x}{2 y}+o\left(\frac{1}{y}\right) \\
P_{\text {share }} & =V_{2}-V_{11}+1=1+\frac{W x}{2 y}+o\left(\frac{1}{y}\right),
\end{aligned}
$$

where $o(1 / y) y \rightarrow 0$ as $y \rightarrow \infty$. The intuition behind the expression for $P_{\max }$ in $(2.2)$ is simple. When $y$ is large, the opportunity cost of searching for an arbitrage position is $W / y+o(1 / y)$. Therefore, the maximum price the incumbent arbitrageur can ask for his existing position is $1+W / y+o(1 / y)$.

The expression for the utility of the incumbent arbitrageur in autarky, $V_{2}$, takes 
also a simple form: $1+W+o(1 / y)$. When the search intensity is very high, the second arbitrage opportunity is readily available after the first opportunity matures. Thus the incumbent arbitrageur constantly invests in one of the two arbitrage opportunities.

If the incumbent arbitrageur gives up autarky then he will have to search for a new opportunity after the current opportunity matures. So he will miss, on average, $x / 2 y$ arbitrage opportunities. Therefore, his utility is reduced by the factor $x W / 2 y$, which results in $V_{11}=1+W-x W / 2 y+o(1 / y)$.

Thus the minimum price at which the incumbent is willing to give up autarky is $P_{\text {share }}=1+x W / 2 y$. Whether it is less than $P_{\max }=1+W / y+o(1 / y)$ depends on the value of $x$. If arbitrage positions must be held for a long time before they pay off, $x<2$, then the congestion effect created by the second arbitrageur is small and it is profitable for the incumbent to sell his existing position despite sharing the arbitrage business in the future. Conversely, if $x>2$ then the incumbent is better off keeping his arbitrage business secret.

\subsection{The Case $x \approx 2 y^{2} \gg y \gg 1$}

This case corresponds to the asymptote of $\psi, x=2 y^{2}$. In this case, using formulas derived in Sections 1.3, 1.4 , and 2 one can show that

$$
\begin{aligned}
P_{\max } & =1+\frac{W}{x}+o\left(\frac{1}{x}\right) \\
V_{2} & =1+W\left(\frac{2 y+\frac{3}{2}}{x}-3\left(\frac{y}{x}\right)^{2}\right)+o\left(\frac{1}{x}\right), \\
V_{11} & =1+W\left(\frac{2 y+1}{x}-\left(\frac{2 y}{x}\right)^{2}\right)+o\left(\frac{1}{x}\right), \\
P_{\text {share }} & =1+\frac{W}{2 x}\left(1+\frac{2 y^{2}}{x}\right)+o\left(\frac{1}{x}\right),
\end{aligned}
$$

where $o(1 / x) x \rightarrow 0$ as $x \rightarrow \infty$. The expression for $P_{\max }$ in (2.6) is again simple to interpret. By not buying the existing position of the incumbent when $x \gg y$, the second arbitrageur forgoes the associated payoff maturing at the expected date $1 / x$. Therefore, the maximum price the incumbent arbitrageur can ask for his existing position is $1+W / x+o(1 / x)$.

To understand the expressions for $V_{2}$ and $V_{11}$ we rewrite equations (2.7) and (2.8) 
as

$$
\begin{aligned}
V_{2} & =1+\underbrace{\frac{W}{x}+\frac{W}{2 x}}_{A}+\underbrace{W\left(\frac{2 y}{x}-3\left(\frac{y}{x}\right)^{2}\right)}_{C}+o\left(\frac{1}{x}\right), \\
V_{11} & =1+\underbrace{\frac{W}{x}}_{B}+\underbrace{W\left(\frac{2 y}{x}-\left(\frac{2 y}{x}\right)^{2}\right)}_{D}+o\left(\frac{1}{x}\right) .
\end{aligned}
$$

Terms $A$ and $B$ correspond to the value that the incumbent expects to receive from the already identified opportunities in autarky and in the case in which he shares the arbitrage business with the other arbitrageur. Terms $C$ and $D$ represent the value from the subsequent opportunities that are yet to open up and be discovered in these two cases.

Consider first terms $A$ and $B$. They both have a term, $W / x$, which is the value of the opportunity in which the incumbent has already invested. In the autarky case, there is also an additional term which represents the expected gain from investing in the identified but not yet funded opportunity. This opportunity outlasts the first opportunity with probability $1 / 2$ after an average $1 / x$ periods. Hence the extra term is $W / 2 x$.

The analysis of the expected value of future opportunities to be discovered is more subtle. Recall that in the $M=N=1$ case in section 1.2 , the present value of future arbitrage opportunities is

$$
W_{0}=\frac{y W}{1+x+y}
$$

which in this limiting case becomes

$$
W_{0}=W\left(\frac{y}{x}-\left(\frac{y}{x}\right)^{2}\right)+o\left(\frac{1}{x}\right) .
$$

The first-order term, $y / x$, is the average number of times the search process is conclusive over the lifetime of an arbitrage opportunity. The second-order term, $-\left(\frac{y}{x}\right)^{2}$, corrects for the fact that an opportunity is no longer to be found once it is already discovered. Thus there are decreasing returns to search. Because the number of opportunities to be discovered is fixed, the additional returns from searching more intensively become small when $y$ becomes large and virtually all opportunities are seized.

In the case in which the incumbent shares the arbitrage business, each future opportunity is searched for with intensity $2 \lambda$. Thus, (2.12) implies that for each arbitrageur, 
the expected gain from opportunities that are yet to be discovered is

$$
W\left(\frac{2 y}{x}-\left(\frac{2 y}{x}\right)^{2}\right)
$$

which is the term $D$ in (2.11).

In autarky, the expected gain from future opportunities is

$$
2 W\left[\frac{y}{x}-\left(\frac{y}{x}\right)^{2}\right]-W\left(\frac{y}{x}\right)^{2} .
$$

The first term reflects the fact that there are two opportunities available. The second term, $-W\left(\frac{y}{x}\right)^{2}$, is due to the capacity constraints: When both opportunities are discovered, only one of them can be exploited. Comparing (2.13) and (2.14), one can now see that by sharing the arbitrage business, the incumbent gains $(y / x)^{2}$ by relaxing capacity constraints, but loses $2(y / x)^{2}$. This is the cost of doubling the search intensity and splitting the resulting value in the presence of decreasing returns to search.

Given the expressions for $V_{2}$ and $V_{11}$, the minimum price at which the incumbent is willing to give up autarky is

$$
P_{\text {share }}=1+\frac{W}{2 x}+W\left(\frac{y}{x}\right)^{2}+o\left(\frac{1}{x}\right)
$$

Comparing it to the $P_{\max }$ in (2.6), one can see that the incumbent is indifferent between keeping the arbitrage business secret and sharing it with the other arbitrageur when $x=2 y^{2}$.

We can summarize the findings of Sections 2.1 and 2.2 as follows. The deliberate limits to arbitrage are more likely to occur when arbitrage opportunities are neither too quick nor too slow to mature. For a fixed $y$, the impact of the duration parameter $x$ is twofold. On the one hand, when arbitrage opportunities pay off more slowly, the costs of future competition become smaller because they occur only after the currently found and exploited opportunities pay off. This reduces the costs of knowledge sharing generated from the sale of the already established arbitrage position. On the other hand, when opportunities are slower to mature, these more remote costs are also more important when they occur. The congestion costs induced by competing arbitrageurs are higher in this case because arbitrageurs exhaust all the trading opportunities open at a given date more frequently. This renders knowledge sharing more costly. Which effect overcomes the other depends on the parameters

When arbitrage opportunities are easier to find, the range of maturities of arbitrage trades for which deliberate limits to arbitrage arise is larger. This happens because a 
more efficient search technology both makes it harder for the incumbent arbitrageur to realize the full value from the sale of the existing positions and increases the effect of future competition.

\section{Extensions}

This paper sheds light on the costs and benefits from sharing proprietary arbitrage information in a fully dynamic, yet analytically tractable setup. Tractability owes to some simplifying assumptions that we discuss in this section.

First, it would be easy to add the feature that a secretive arbitrageur cannot remain the sole owner of his search technology forever. Arbitrage ideas can ultimately become public knowledge. For instance, prime brokers can ultimately be able to understand the ideas behind their customers' trades, and copy them. In our setup, if search technologies were to become public information after some random time, it would reduce the benefits from autarky, and make sharing the arbitrage business more likely.

Second, the assumption that the description of a position is bundled with the perfect communication of the underlying knowledge is also for tractability, and could be relaxed. One could for example assume that such communication occurs only with some probability, or that the search technology acquired by the buyer is less efficient than that of the seller. This would make communicating arbitrage positions more likely. More generally, it would also be interesting to introduce learning-by-doing, whereby arbitrageurs' searching skills increase as they complete more trades.

Third, the limits-to-arbitrage literature often studies a situation in which an arbitrageur is subject to non-insurable liquidity shocks, for example, because of outflows of 'dumb' money after a negative performance shock. In our paper, identification of the second opportunity after having invested in the first one - and thus being capacityconstrained - can also be viewed as an (endogenous) increase in the cost of holding an illiquid position. It would be straightforward to have additional exogenous liquidity shocks in this environment, as, e.g., in Duffie, Garleanu, and Pedersen (2005).

Fourth, an interesting, and more involved, extension of our model would consist in an explicit modelling of the process through which arbitrageurs' trades eliminate mis-

pricings. Presumably, the returns to arbitrage opportunities, now characterized by the exogenous parameters $R$ and $x$, would then depend on the equilibrium number of arbitrageurs. This would ultimately shed light on how the nature of arbitrage knowledge determines market efficiency.

Finally, the explicit modelling of liquidity shocks and of the process through which arbitrageurs' trades eliminate mispricings would allow one to incorporate in the analysis 
the possibility of predatory trading by sophisticated investors. Predatory trading is studied in Brunnermeier and Pedersen (2005) and is commonly viewed by market participants as another rationale for keeping arbitrage trades secret.

These possible extensions suggest that the framework developed in this paper is versatile. The stripped-down version of the model that we use illustrates the broad trade-offs associated with the dissemination of arbitrage knowledge. We hope that this sets a useful starting point for future research on this question. Many aspects of the nature of arbitrage knowledge remain to be explored in order to better understand its role in the persistence of limits to arbitrage in well-developed, sophisticated financial systems.

\section{References}

[1] Anton, J. and Yao, D., "The Sale of Ideas: Strategic Disclosure, Property Rights, and Contracting," The Review of Economic Studies, 69, 513-531 (2002)

[2] Arrow, K., "Economic Welfare and the Allocation of Resources for Inventions," in R. Nelson (ed.) The Rate and Direction of Inventive Activity: Economic and Social Factors, Princeton University Press, (1962)

[3] Bhattacharya, S. and Ritter, J., "Innovation and Communication: Signalling with Partial Disclosure," The Review of Economic Studies, 50, 331-346 (1983)

[4] Barberis N. and Thaler R., "A survey of Behavioral Finance", Handbook of the Economics of Finance, (2003)

[5] Brunnermeier, M., and Pedersen, L., "Predatory Trading," The Journal of Finance, $60,1825-1863$ (2005)

[6] Brunnermeier, M., and Pedersen, L., "Market Liquidity and Funding Liquidity," The Review of Financial Studies, 22, 2201-2238 (2009)

[7] Dow, J., and Gorton, G., "Arbitrage Chains," The Journal of Finance, 49 (3), 819-849 (1994)

[8] Duffie, D., Garleanu, N., and Pedersen L., "Over-the-Counter Markets," Econometrica, $73,1815-1847$ (2005)

[9] Friedman, M., "Essays in Positive Economics," University of Chicago Press (1953)

[10] Goldin, C., and Katz, L. F. ,"Transitions: Career and Family Lifecycles of the Educational Elite," AEA Papers and Proceedings, 363-69 (2008) 
[11] Gromb, D., and Vayanos, D., "Limits of Arbitrage," Annual Review of Financial Economics, 2, 251-275 (2010)

[12] Gromb, D., and Vayanos, D., "The Dynamics of Financially Constrained Arbitrage," working paper (2011)

[13] He, Z., and Krishnamurthy, A., "A Model of Capital and Crises," forthcoming, The Review of Economic Studies.

[14] Kaplan, S.N., and Rauh, J., "Wall Street and Main Street: What Contributes to the Rise in the Highest Incomes?" Review of Financial Studies 23(3), 1004-1050 (2010).

[15] Kondo, J. and Papanikolaou, D., "Financial Relationships and The Limits to Arbitrage," working paper (2005)

[16] Kondor, P., "Risk in Dynamic Arbitrage: Price Effects of Convergence Trading," The Journal of Finance, 64(2), 638-658 (2009)

[17] Lamont, O. and Thaler R., "The Law of One Price in Financial Markets," Journal of Economic Perspectives, 17(4), 191202 (2003)

[18] Philippon, T., and Reshef, A., "Wages and Human Capital in the U.S. Finance Industry: 1909-2006," working paper (2011)

[19] Shleifer A., and Vishny R., "The Limits of Arbitrage," The Journal of Finance, 52 (1), 35-55 (1997)

\section{Appendix}

\section{Proof of Proposition 1}

Straightforward computations yield the expression for $\Phi$. Further,

$$
\begin{aligned}
& \varphi_{4}(x)<0 \text { for } x \in[0,2), \\
& \varphi_{3}(x)<0 \text { for } x \in[0,3.59), \\
& \varphi_{2}(x)<0 \text { for } x \in[0,6.38), \\
& \varphi_{1}(x)<0 \text { for } x>0, \\
& \varphi_{0}(x)<0 \text { for } x>0 .
\end{aligned}
$$

This implies that $\Phi(x, y)<0$ as soon as $x \leq 2$, and that there exists $y>0$ such that $\Phi(x, y)=0$ for each $x>2$. To see that such a $y$ is unique for every $x$, remind that the 
maximum number of positive zeros of a polynomial function is given by the number of changes in the sign of the coefficients, and that the actual number of zeros may differ from this maximum by an even number. 